OPEN ACCESS

Edited by:

Bahar Güntekin,

School of International Medicine,

Istanbul Medipol University, Turkey

Reviewed by:

Roberta Lizio,

Sapienza Università di Roma, Italy

Lewis A. Wheaton,

Georgia Institute of Technology,

United States

*Correspondence:

Zhi-Peng Liu

Izpeng67@163.com

Tao Yin

bme500@163.com

Received: 20 July 2017 Accepted: 13 November 2017 Published: 29 November 2017

Citation:

Jin J-N, Wang $X, L i Y$, Jin F, LiU Z-P and Yin T (2017) The Effects of rTMS

Combined with Motor Training on

Functional Connectivity in Alpha

Frequency Band.

Front. Behav. Neurosci. 11:234.

doi: 10.3389/fnbeh.2017.00234

\section{The Effects of rTMS Combined with Motor Training on Functional Connectivity in Alpha Frequency Band}

\author{
Jing-Na Jin ${ }^{1,2}$, Xin Wang ${ }^{1}$, Ying Li ${ }^{1}$, Fang Jin ${ }^{1}$, Zhi-Peng Liu ${ }^{1,2 *}$ and Tao Yin ${ }^{1,2 *}$ \\ ${ }^{1}$ Institute of Biomedical Engineering, Chinese Academy of Medical Sciences \& Peking Union Medical College, Tianjin, China, \\ ${ }^{2}$ Neuroscience Center, Chinese Academy of Medical Sciences, Beijing, China
}

It has recently been reported that repetitive transcranial magnetic stimulation combined with motor training (rTMS-MT) could improve motor function in post-stroke patients. However, the effects of rTMS-MT on cortical function using functional connectivity and graph theoretical analysis remain unclear. Ten healthy subjects were recruited to receive rTMS immediately before application of MT. Low frequency rTMS was delivered to the dominant hemisphere and non-dominant hand performed MT over 14 days. The reaction time of Nine-Hole Peg Test and electroencephalography (EEG) in resting condition with eyes closed were recorded before and after rTMS-MT. Functional connectivity was assessed by phase synchronization index (PSI), and subsequently thresholded to construct undirected graphs in alpha frequency band $(8-13 \mathrm{~Hz})$. We found a significant decrease in reaction time after rTMS-MT. The functional connectivity between the parietal and frontal cortex, and the graph theory statistics of node degree and efficiency in the parietal cortex increased. Besides the functional connectivity between premotor and frontal cortex, the degree and efficiency of premotor cortex showed opposite results. In addition, the number of connections significantly increased within inter-hemispheres and inter-regions. In conclusion, this study could be helpful in our understanding of how rTMS-MT modulates brain activity. The methods and results in this study could be taken as reference in future studies of the effects of rTMS-MT in stroke patients.

Keywords: repetitive transcranial magnetic stimulation, motor training, phase synchronization index, functional connectivity, graph theory

\section{INTRODUCTION}

Motor training (MT) can influence cortical activity related to motor task, and is a major means of improving limb movement function, especially in people with motor dysfunction, such as stroke patients (Van et al., 2001; Higgins et al., 2006). However, the motor functional outcomes induced by MT often have limitations. Transcranial magnetic stimulation (TMS) is a non-invasive brain stimulation method that can alter cortical excitability via application of time-varying magnetic field. Repetitive TMS (rTMS) is one TMS pattern that produces serial outputs of TMS stimuli pulses. rTMS can modulate cortical activity. The changes in cortical activity can persist long after the end of stimulation (Thut and Pascual-Leone, 2010) to influence motor and cognitive functions (Baeken et al., 2012; Turriziani et al., 2012; D’Agata et al., 2016). Therefore, rTMS has been proposed as 
a potential method to improve motor functions in stroke patients (Fregni et al., 2006; Conforto et al., 2012; Brodie et al., 2014).

Recently, a majority of studies has found the combination protocol of rTMS-MT to improve motor functions of the paretic upper limb to an extent that was unattainable by either rTMS or MT alone (Bolognini et al., 2009; Emara et al., 2010; Avenanti et al., 2012; Kakuda et al., 2012, 2016; LüdemannPodubecká et al., 2015). Avenanti et al. (2012) studied the long-term behavioral effect of rTMS-MT in chronic stroke, and found that receiving MT prior to the rTMS was optimal in boosting functional outcomes. Furthermore, although there were three combination patterns for rTMS to improve motor functional outcomes, including downregulation of excitability by low frequency rTMS over contralesional hemisphere $(\leq 1 \mathrm{~Hz})$, upregulation of excitability by high frequency rTMS over lesional hemisphere $(\geq 5 \mathrm{~Hz})$, and bihemispheric regulation by low frequency rTMS over contralesional hemisphere associated with high frequency rTMS over the lesional hemisphere, the first one was the most popular method used in stroke patients. One reason for this choice might be that low frequency rTMS was safer and had lower probability of epileptic complication. Another reason might be that the effects of low frequency rTMS stimulation of the contralesional hemisphere on brain activity was slightly affected by changes in the brain induced by stroke.

Although many studies have shown that rTMS-MT could improve motor performance in stroke patients, the impacts of rTMS-MT on brain activity remain to be fully understood. Takekawa et al. (2014) studied the effects of rTMS-MT on regional brain perfusion using single-photon emission computed tomography (SPECT) in chronic stroke patients, and found changes in asymmetry index in the superior and middle frontal areas, which correlated significantly and negatively with changes in Fugl-Meyer assessment (FMA) score. In another study, the authors measured F-wave parameter to evaluate the impact of rTMS-MT on motor neural excitability (Kondo et al., 2015). However, studies that tested the effects of rTMS-MT on functional network are scarce.

The human cerebral cortex exhibits specific functional interconnection patterns that link all brain regions. The linking patterns are neither completely regular nor random, i.e., the human brain integrates both localized and segregated information processing (Sporns et al., 2004), and can be modulated by circumstances and external stimuli. A number of studies have demonstrated that rTMS protocols could alter the activity and function of targeted brain region as well as its related remote regions (Jing and Takigawa, 2000; Plewnia et al., 2008; Grefkes et al., 2010). The activity of many cortices related to motor function, such as premotor, primary motor, and posterior parietal cortex can also be changed by MT (Youssofzadeh et al., 2016; De Vico Fallani et al., 2017). Instead of local regional activity, functional connectivity, which can be obtained through analysis of inter-regional coupling, reflects the functional interactions between the underlying brain regions (Ward and Cohen, 2004; Grefkes et al., 2010). Therefore, we can speculate that rTMS and MT could modulate brain functional connectivity. However, there are limited studies that have analyzed the modulation of rTMS-MT on functional connectivity.
Brain activity in the resting state with eyes closed is the baseline status of the human brain that is independent of taskrelated brain functions. The study of resting state neural activity is an alternative approach to task related regional activity and likely provides additional information on the neurobiological mechanisms of specific brain systems (Fox and Raichle, 2007; Van Dijk et al., 2010). Neural oscillation at alpha frequency band (8$13 \mathrm{~Hz}$ ) is the main rhythm in the resting state and constitutes an important neural substrate for cognition and action. This oscillation is supposed to be able to predict the efficiency of neural cognitive-motor processes when the subject is involved in demanding tasks, in eyes closed resting state condition (Babiloni et al., 2010). Thus, it is critical to study the changes of alpha band neural activity in the resting state in order to understand brain responses induced by rTMS-MT.

Studies of complex human brain networks using graph theory as a mathematical model have attracted much interest in recent years (Stam and Reijneveld, 2007; Stern, 2013). This approach consists of a set of elements (nodes) and connections (edges) that interconnect the nodes of the graph. In our study, each node was represented by an electroencephalography (EEG) electrode and the edges by functionally connected brain regions. This approach can be used to examine changes in functional architectural connectivity of the brain, which result from diseases and external stimuli (Ward and Cohen, 2004; Grefkes et al., 2010). The results of recent studies suggest that functional disconnection between distant brain areas could at least partly explain many brain dysfunctions, such as Alzheimer's disease (AD) and stroke (Stam et al., 2007a,b). Therefore, brain functional connectivity and graph theoretical analysis may be combined to achieve a better insight into the impact of rTMS-MT on cortical functions.

In order to understand the impact of rTMS-MT on brain activity, we recruited healthy subjects and evaluated whether functional cortical architecture at alpha frequency band in the resting state changed. To achieve this, we first tested their behavioral performances before and after rTMS-MT. Then, we recorded 60-channels EEG before and after rTMS-MT in healthy volunteers who were in resting state with eyes closed. Correlation matrices were based on calculation of the phase synchronization index (PSI) between all pair wise combinations of the 60 EEG channels and subsequently undirected graphs were built. Finally, we calculated the relationship between functional connectivity and behavioral data by Pearson's correlation. Our methods and results could be helpful in future for studying the effects of rTMS-MT in stroke patients.

\section{MATERIALS AND METHODS}

\section{Subjects}

The study involved 10 healthy volunteers (10 men; mean age $23.4 \pm 2.1$ years). None of them suffered from any significant neurological disorder or had any implanted metallic electrical devices or took any medication in the 2 weeks before their participation in the experiment. All subjects were right handed according to the Edinburgh handedness inventory. The study was performed according to the Declaration of Helsinki and approved by the Ethics Committee of Institute of Biomedical 
Engineering, Chinese Academy of Medical Sciences \& Peking Union Medical College. All subjects provided informed consent prior to inclusion in the study.

\section{Procedure}

In our study, the purpose of rTMS-MT was to improve the motor activity of non-dominant hand. The main reason was that, compared with dominant hand, the non-dominant hand was not usually used in daily day and couldn't perform lots of movements flexibly. We selected three MTs which were unfamiliar to nondominant hand, and detected the improvement of non-dominant hand induced by rTMS-MT. In other words, the non-dominant hand of healthy person was analogied to the lesional-hand of stroke in our study.

In general, the rTMS-MT was based on a model of interhemispheric competition model in stroke (Farzan et al., 2010). Up-regulating excitability of the lesioned motor cortex or down-regulating excitability in the intact motor cortex could improve the motor performance. Therefore, in our study, in order to achieve improved motor functions of the non-dominant hand, low frequency rTMS was first delivered to the dominant hemisphere to downregulate its excitability, followed by training of the non-dominant hand intended to upregulate the excitability of the non-dominant hemisphere (Avenanti et al., 2012). Figure 1 shows the experimental paradigm. The subjects performed rTMS-MT for 14 days. The Nine-Hole Peg Test was performed to assess the dexterity of bilateral hands. The subjects had to complete the Nine-Hole Peg Test as quickly as possible. The reaction time was recorded, and resting EEG with eyes closed was obtained before and after rTMS-ML.

\section{Repetitive Transcranial Magnetic Stimulation}

rTMS was delivered through a figure-eight shaped coil $(70 \mathrm{~mm}$ standard coil, Mastic, Whiteland, UK) connected to a Mastic Rapid2 stimulator (Mastic, Whitland, UK). In order to precisely stimulate the target brain area (primary motor cortex), we used a frameless stereotactic neuro-navigation system (Brainsight, Rogue Inc., UK). All stimulation was applied over the left primary motor cortex (dominant hemisphere). The coil was placed tangentially to the scalp with the handle pointing backwards and laterally at a $45^{\circ}$ angle away from the midline. The primary motor cortex was individually localized for each participant based on the optimal position for eliciting motor-evoked potentials (MEPs) in the right abductor pollicis brevis (APB). The individual resting motor threshold (RMT) was defined as the minimum stimulus intensity that could produce an MEP of at least $50 \mu \mathrm{V}$ in at least five out of 10 consecutive trials.

Each subject received 10 sessions of rTMS at a frequency of $1 \mathrm{~Hz}$ over the 14 days, excluding Sundays. The stimulation target was the left hemisphere over the primary motor cortex corresponding to the "hot spot" for stimulation of the APB cortical area representation as defined during RMT determination. One rTMS session consisted of 1,200 pulses, lasting $20 \mathrm{~min}$. The intensity of stimulation was set at 90\% RMT.

\section{Motor Training}

The MT consisted of 3 standardized exercises designed to improve dexterity of the non-dominant hand. The first exercise, which lasted 10 min each day, was to turn coins over using the index finger and thumb. The second was to put a knot around a screw by holding the knot and screw with the non-dominant and dominant hands, respectively. The third, which lasted $40 \mathrm{~min}$ each day, was to write letters. The duration of MT was $60 \mathrm{~min}$ each day.

\section{EEG Recording}

Electroencephalography (EEG) was recorded for two and a half minutes before and after rTMS-MT in an awake, resting state during which subjects kept their eyes closed. We chose to test subjects with eyes closed to reduce the presence of eye movement and muscle artifacts. Electrode montage and placement was recording to the international 10/10 system. EEG signals were acquired through a 64-channel synamps2 EEG system (Neuroscan, Compumedics, USA). A 64-channel EEG cap was positioned on subjects' head. The reference electrode was at $\mathrm{AFz}$ site, whereas the ground electrode was at $\mathrm{FCz}$ site. The impedance for all electrodes was kept below $5 \mathrm{k} \Omega$. The activities in the right eye vertical and horizontal electroculogram (vEOG) were registered from two surface electrodes. The EEG data was sampled at a frequency of $1,000 \mathrm{~Hz}$ and subsequently processed offline.

\section{Phase Synchronization Index}

Phase synchronization index (PSI) can quantify neural signal interaction between brain regions, but neglect the effect of instantaneous amplitude. This method has been proven effective in inferring functional connectivity between neural oscillations (Varela et al., 2001; Ito et al., 2005; Wu et al., 2011).

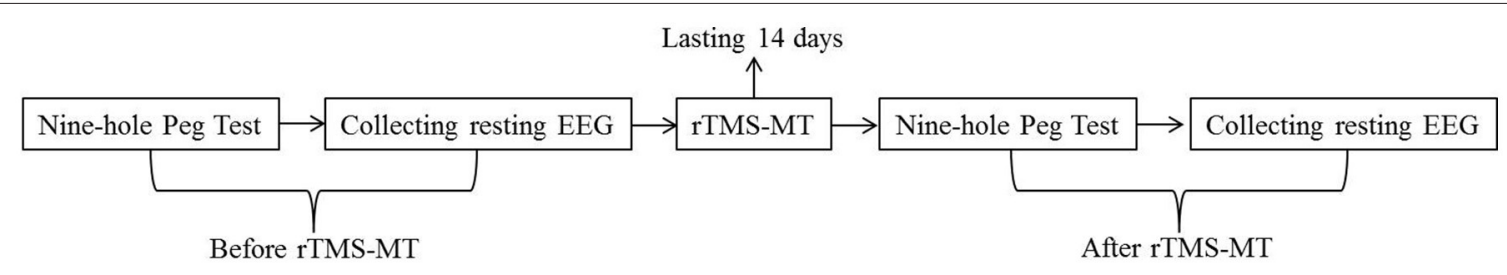

FIGURE 1 | Experimental paradigm. Low frequency rTMS was first delivered to the dominant hemisphere, followed by training of the non-dominant hand, which lasted 14 days. Nine-Hole Peg Test and resting state EEG with eyes closed were recorded before and after rTMS-MT. 
Given an EEG signal $x(t)$, its analytical signal is defined as:

$$
\mathrm{Z}_{\mathrm{x}}(t)=\mathrm{x}(t)+\mathrm{i} \overline{\mathrm{x}}(t)=\mathrm{A}_{\mathrm{x}}^{\mathrm{H}}(t) \mathrm{e}^{i \Phi_{\mathrm{x}}^{\mathrm{H}}(t)}
$$

Where

$$
\bar{x}(t)=H x(t)=\frac{1}{\pi} \int_{-\infty}^{+\infty} \frac{x\left(t^{\prime}\right)}{t-t^{\prime}} d\left(t^{\prime}\right)
$$

is the Hilbert transformation of signal $x(t) . \mathrm{A}_{\mathrm{X}}^{\mathrm{H}}(t)$ and $\Phi_{\mathrm{X}}^{\mathrm{H}}(t)$ are the instantaneous amplitude and phase of signal $\mathrm{x}(t)$, respectively. Let $\Phi_{\mathrm{x}}^{\mathrm{H}}(t)$ and $\Phi_{\mathrm{y}}^{\mathrm{H}}(t)$ denote the cumulative instantaneous phases of two coupled signals, respectively. If $\Phi_{\mathrm{x}}^{\mathrm{H}}(t)$ and $\Phi_{\mathrm{y}}^{\mathrm{H}}(t)$ satisfy.

$$
\Phi_{\mathrm{xy}}^{\mathrm{H}}(t) \equiv\left|n \Phi_{\mathrm{x}}^{\mathrm{H}}(t)-m \Phi_{\mathrm{y}}^{\mathrm{H}}(t)\right|<\text { const. },
$$

then the two signals $\mathrm{x}(t)$ and $\mathrm{y}(t)$ are said to be in $n: m$ phase synchronization, where const. is a constant, and $n$ and $m$ are positive integers. Equation (3) implies that the $n: m$ instantaneous phase difference of signals $\mathrm{x}(t)$ and $\mathrm{y}(t)$ is bounded. As mentioned by a number of authors (Quiroga et al., 2000; Quian Quiroga et al., 2002; Angelini et al., 2004; David et al., 2004), 1:1 phase synchronization is the case for most neurophysiological signals. The PSI is defined as:

$$
\lambda_{\mathrm{H}}=\left|\left\langle\mathrm{e}^{\mathrm{i} \Phi_{\mathrm{xy}}^{\mathrm{H}}(t)}\right\rangle_{t}\right|=\sqrt{\left\langle\cos \Phi_{\mathrm{xy}}^{\mathrm{H}}(t)\right\rangle_{t}^{2}+\left\langle\sin \Phi_{\mathrm{xy}}^{\mathrm{H}}(t)\right\rangle_{t}^{2}},
$$

where $\langle$.$\rangle denotes the average over time. Note that the value$ of $\lambda_{\mathrm{H}}$ is among $[0,1]$ with $\lambda_{\mathrm{H}}=1$ implying perfect phase synchronization, and $\lambda_{\mathrm{H}}=0$ indicating no phase synchronization at all.

\section{Generating Synchronization Matrices and Graph Theory Analysis}

To evaluate changes in network topologies induced by rTMS-MT, we constructed undirected graphs before and after rTMS-MT. There are 60-channels EEG signals for each subject. For each subject, we could obtain a 60-by-60 matrix for all 60 channels in pairs. Then, for each individual data set, a connectivity graph was formed consisting of 60 nodes (EEG channels) and a set of undirected edges, A (functional connectivity) obtained by applying a correlation threshold, T to the PSI matrix:

$$
A_{i j}=\left\{\begin{array}{ll}
1 & \lambda_{\mathrm{H}} \geq T \\
0 & \lambda_{\mathrm{H}}<T
\end{array}(\mathrm{i}, \mathrm{j}=1,2, . . .60)\right.
$$

Hence, if the PSI value between a pair of brain regions $i$ and $j$ was greater than the given value $\mathrm{T}$, an edge was said to exist.

In our study, the threshold was set independently before and after rTMS-MT to produce the same mean connectivity degree in each period (over a range of mean connectivity degrees) for each subject and for the grand average across subjects (fixeddensity analyses). The fixed-density analyses were performed systematically over a range of thresholds, resulting in networks with average connection densities ranging from two up to 20 connections per node.

The three most commonly evaluated graph theory statistics include the node degree, clustering coefficient, and efficiency. The node degree is defined as the number of edges one node connects with other nodes. The greater the node degree, the more edges this node has, and therefore could exert greater influence on the network. The degree, $\mathrm{D}$ of node $\mathrm{i}$ is defined as:

$$
D_{i}=\sum_{j=1}^{N} a_{i j}
$$

The clustering coefficient evaluates the proportion of neighboring vertices (i.e., vertices directly connected to the node in question) that are connected to each other. Networks with higher clustering are said to have greater local efficiency of information processing and robustness. The clustering coefficient of node $i$ is defined as.

$$
C_{i}=\frac{2 E_{i}}{k_{i}\left(k_{i}-1\right)}
$$

Where $k_{i}$ is the number of neighboring nodes connected with node $i$, and $k_{i}\left(k_{i}-1\right) / 2$ denotes all possible edges among neighbor nodes of node $i$.

Node efficiency is the average of the inverse of the shortest path length, and reflects the efficiency of information transmission. The greater the node efficiency, the more efficient the information transfer. The efficiency of node $i$ is defined as:

$$
E_{\text {glob }}=\frac{1}{N(N-1)} \sum_{i, j \subseteq V, i \neq j} \frac{1}{d_{i j}}
$$

\section{Partition of All Electrodes}

To quantitatively examine patterns of changes in functional connectivity during rTMS-MT, we grouped connections along the anterior-posterior and right-left axes, and performed statistical tests on these groups. Figure 2 shows the schematic diagram of partition for all the electrodes. In the anteriorposterior analysis (Figure 2A), the EEG channels were first partitioned into anterior, central, or posterior regions. The anterior region consisted of Fp1, Fp2, F7, F3, Fz, F4, F8, FC5, FC1, FC2, and FC6. The central region consisted of T7, C5, C3, C1, $\mathrm{Cz}, \mathrm{C} 2, \mathrm{C} 4$, and $\mathrm{T} 8$. The posterior region consisted of CP5, CP1, CP2, CP6, P7, P3, Pz, P4, P8, O1, Oz, and O2. If both electrodes were anterior, or if one was anterior and the other central, then the connection between the two was designated as "anterior." If both electrodes were posterior, or if one was posterior and the other central, the connection was "posterior." Connections where one electrode was from the anterior region and the other from the posterior region were defined as "interregional." Connections between two central electrodes were ignored during subsequent analyses.

To analyze changes in connectivity induced by rTMS-MT within a hemisphere, EEG channels were first partitioned 
into left hemispheric, right hemispheric, and midline subsets according to standard EEG convention (left hemisphericFp1, F7, F3, FC5, FC1, T7, C5, C3, C1, CP5, CP1, P7, $\mathrm{P} 3$, and $\mathrm{O} 1$; right hemispheric-Fp2, F8, F4, FC6, FC2, T8, $\mathrm{C} 4, \mathrm{C} 2, \mathrm{CP} 6, \mathrm{CP} 2, \mathrm{P} 8, \mathrm{P} 4$, and $\mathrm{O} 2$; midline-Fz, Cz, and $\mathrm{Oz}$; Figure 2B). If both electrodes were right hemispheric, or if one was right hemispheric and the other midline, the connection between the two was designated as "right intra-hemispheric" connection. If both electrodes were left hemispheric, or if one was left hemispheric and the other midline, the connection was designated as a "left intra-hemispheric" connection. If one electrode was right hemispheric and the other left hemispheric, the connection was designated as "interhemispheric." Connections between two midline electrodes were ignored. Subsequent statistical analysis was conducted following the same approach as described above for the connections grouped according to regions.

\section{Data Processing and Statistical Analysis}

Electroencephalography (EEG) data was processed offline using Matlab (version 10.0) and EEGLAB toolbox (version 13.0). The preprocessing stage of the EEG signals is required before further analysis. $1 \mathrm{~min}$ data without apparent artifacts (such as EMG and visible drift) were selected manually from each volunteer's EEG recording. The data were first down-sampled from 1,000 to $250 \mathrm{~Hz}$. And then all channels were rereferenced to bilateral mastoid. The bandpass filter was used to extract alpha band signals $(8-13 \mathrm{~Hz})$. The alpha band signals were then split into 5 -s, non-overlapping epochs in the following steps of feature extraction. 12 epochs of artifact-free EEG data for each subject were obtained finally.

Statistical differences before and after rTMS-MT were tested with a nonparametric rank sum test. Pearson's correlation was used to assess the relation between PSI and hand dexterity. All data were presented as mean \pm standard deviation. A $p<0.05$ was considered statistically significant, except the PSI functional connectivity $(p<0.005)$. All statistical analyses were performed using matlab software (Mathworks Inc., US).

\section{RESULTS}

\section{Behavioural Results}

The 14-days rTMS-MT resulted in a significant decrease in reaction time for left hand (rTMS-MT group, before: $19.1 \pm$ $1.1 \mathrm{~s}$, after: $17.5 \pm 1.4 \mathrm{~s}, p=0.001$; Figure 3), but not right hand dexterity (before: $17.9 \pm 0.8 \mathrm{~s}$, after: $17.6 \pm 1.6 \mathrm{~s}, p=0.281$; Figure 3). The data suggested a significant improvement of motor performance for the left hand (non-dominant hand).

\section{Functional Connectivity}

A $60 \times 60$ channel matrix, consisting of the PSI values for each electrode pair, was obtained for each subject before and after rTMS-MT, and significant changes in connectivity were assessed for all subjects at a significance level of $p<0.005$. Figure 4A shows the functional connectivity with significant difference before and after rTMS-MT. The results suggest that the functional connectivity in the alpha frequency band was
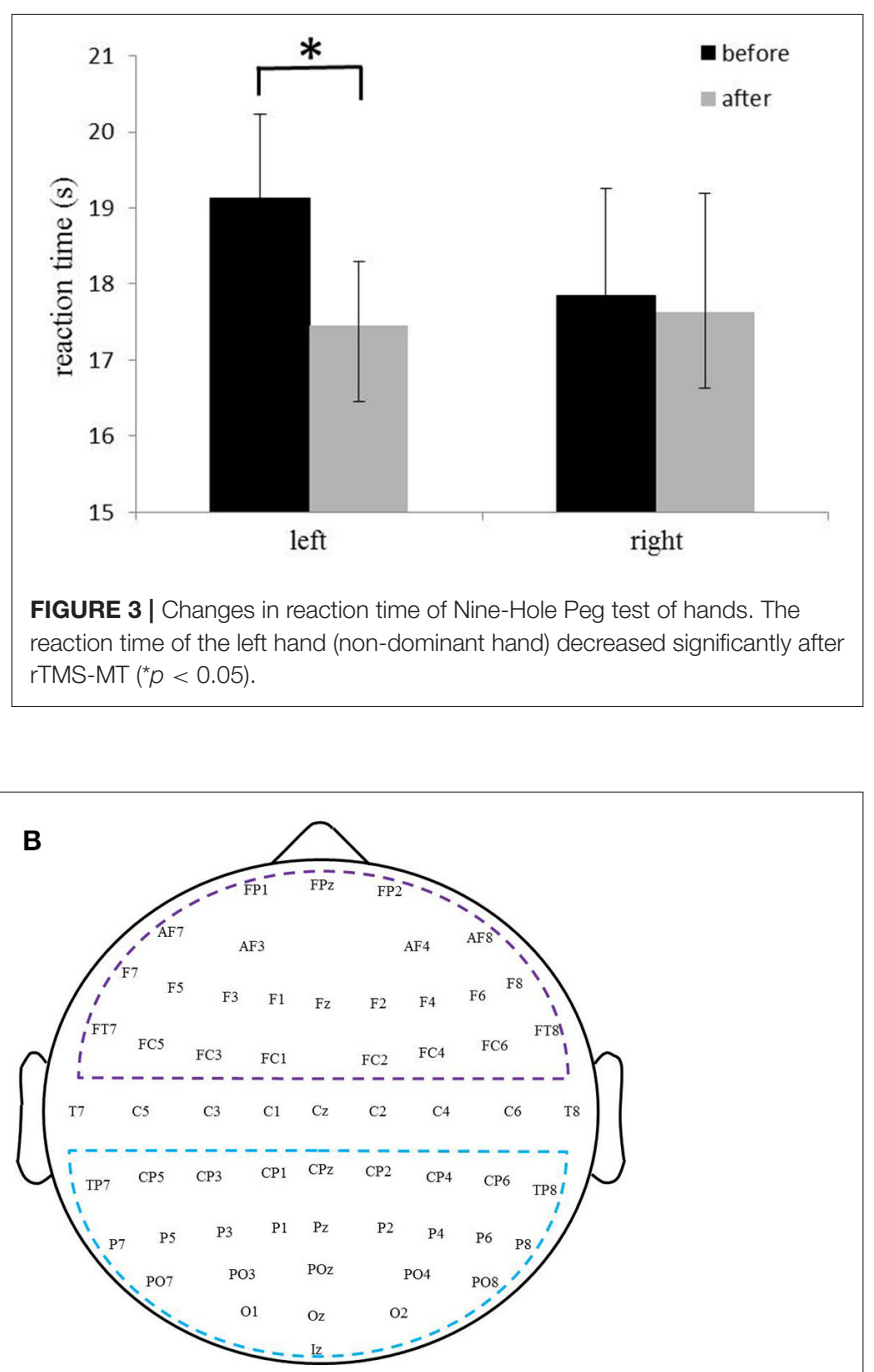

FIGURE 2 | A schematic diagram of partition for all electrodes. (A) Left-right hemispheric. (B) Anterior-posterior region. 

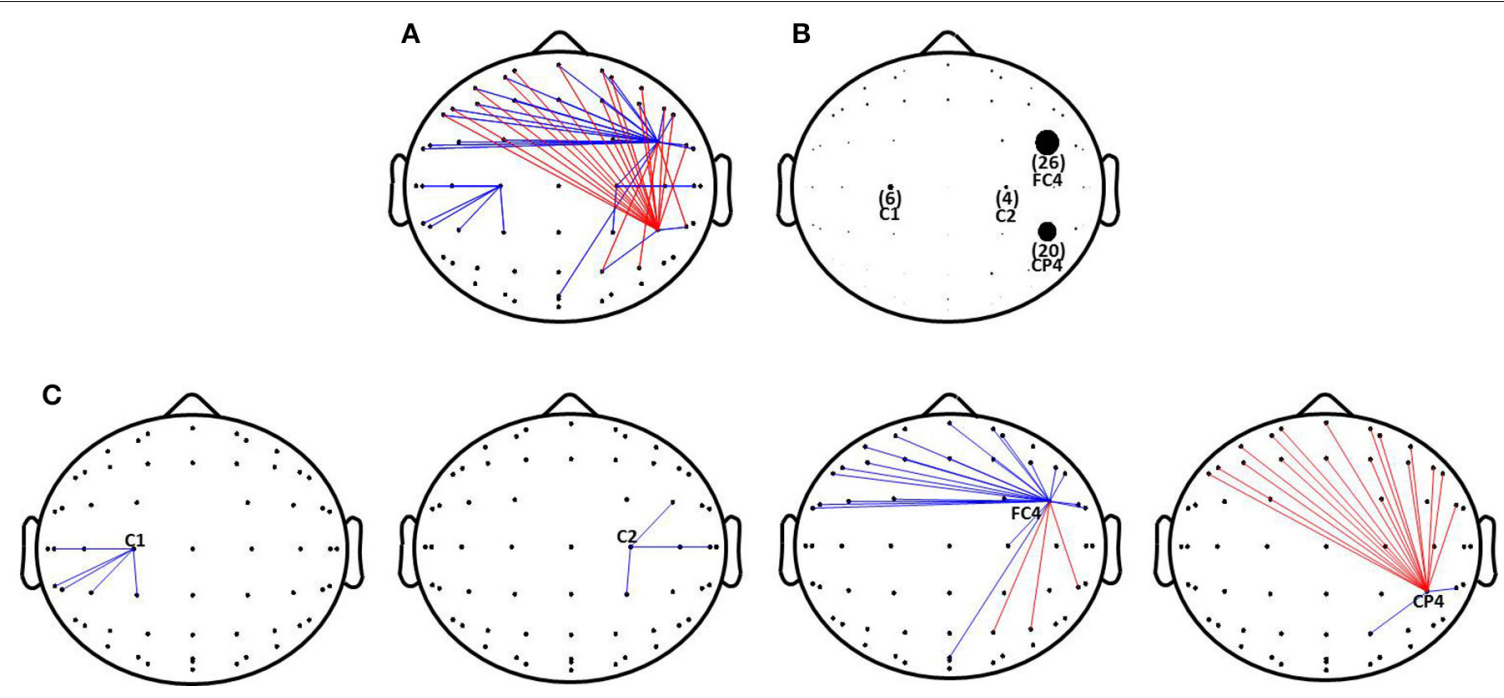

FIGURE 4 | Changes of functional connectivity in alpha frequency band induced by rTMS-MT. Significant decrease and increase in functional connectivity are designated by blue and red lines, respectively ( $\left.{ }^{*} p<0.005\right)$. (A) Changes of functional connectivity. (B) The number of functional connectivity induced by $r$ TMS-MT. (C) Changes of functional connectivity in $\mathrm{C} 1, \mathrm{C} 2, \mathrm{FC} 4$, and CP4 electrodes.

changed by the 14-days rTMS-MT, and the changes were regionspecific.

In order to clearly show the changes in functional connectivity, we calculated the number of connections with significant differences $(p<0.005)$, and expressed them with different-size dots (Figure 4B). rTMS-MT might mainly affect brain regions related to motor function in the right hemisphere (non-dominant hemisphere), especially FC4 and CP4. There was a decrease in strength of functional connectivity between FC4 and the frontal region, and an increase between CP4 and the frontal region (Figure 4C). Furthermore, C1 and C2 were also slightly influenced. There was a decrease between $\mathrm{C} 1, \mathrm{C} 2$, and parietal region of the ipsilateral hemisphere (Figure 4C).

\section{Network Topology}

Figure 5 shows the number of connections in intra-hemispheres, inter-hemispheres, intra-regions, and inter-regions with a mean connectivity degree from 2 to 20 before and after rTMS-MT. It could be observed that the number of connections decreased within intra-hemispheres and intra-regions, and increased within inter-hemispheres and inter-regions in all connectivity degrees. A nonparametric rank sum test was performed $\left({ }^{*} p<0.05\right)$. Significant differences were found within interhemispheres, left intra-hemispheres ( $\mathrm{K}=$ from 12 to 14 ), and inter-regions $(\mathrm{K}=$ from 2 to 20 ) before and after rTMS-MT.

\section{Network Characteristics}

From the results of functional connectivity, it can be inferred that rTMS-MT mainly induced changes at nodes FC4, CP4, C2, and $\mathrm{C} 1$ in alpha band. We calculated the network characteristics including node degree, clustering coefficient, and efficiency of nodes with different connectivity degrees ( $K=$ from 2 to 20 ), and the results are shown in Figure 6. It could be observed that both node degree and efficiency decreased at FC4 $(\mathrm{K}=$ from 2 to 20 ) and $\mathrm{C} 1$ ( $\mathrm{K}=$ from 4 to 12, 20 for node degree; $\mathrm{K}=8$ for node efficiency), but increased significantly at CP4 ( $\mathrm{K}=$ from 2 to 20$)$ in the variable-densities networks. The clustering coefficient was not significantly different before and after rTMS-MT.

\section{Relationship of Functional Connectivity and Behavioral Results}

We calculated the number of significant functional connections between the FC4, CP4, C1, C2, and other electrodes before and after rTMS-MT. The relationship of functional connectivity and reaction time of Nine-Hole Peg Test for the -dominant hand was calculated using Pearson's correlation, and the results are shown in Table 1. We found strong positive correlation between changes in functional connectivity and motor function at FC4 $(r=0.712$, $p=0.048)$. We also found correlations between changes in functional connectivity and motor function at $\mathrm{C} 1(r=0.672$, $p=0.068)$ and $\mathrm{C} 2(r=0.594 p=0.121)$ respectively, even if there were not significant.

\section{DISCUSSION}

It had been reported that rTMS-MT could improve motor performance, however, studies on modulation of rTMS-MT on brain functional connectivity were still scarce. In our study, we evaluated the changes of functional connectivity in alpha frequency band induced by 14-days of rTMS-MT. We found that rTMS-MT significantly improved hand dexterity. The functional connectivity and network characteristics of cortices related to motor function in non-dominant hemisphere changed, and the number of connections mainly increased within 

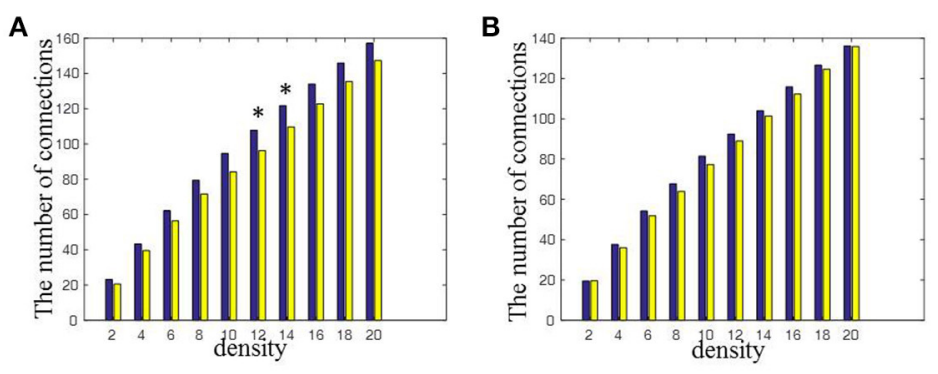

D

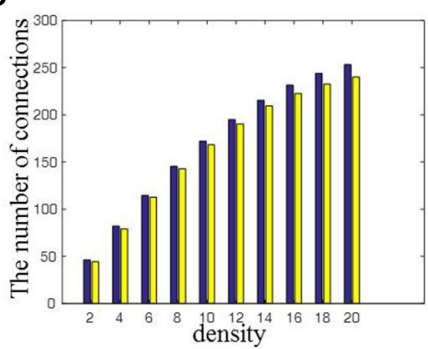

E

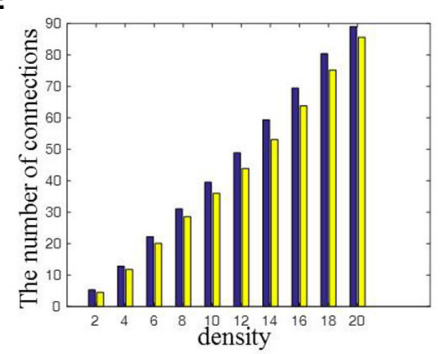

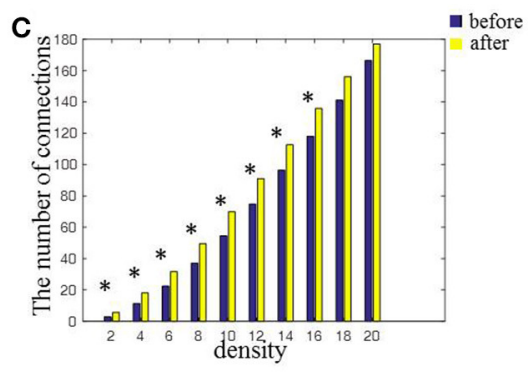

$\mathbf{F}$

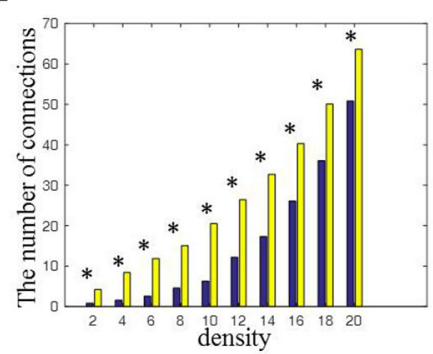

FIGURE 5 | The changes in connectivity induced by rTMS-MT for alpha frequency band between intra-hemispheric regions, inter-hemispheric regions, intra-regions, and inter-regions ( $\left.{ }^{*} p<0.05\right)$. (A) Left-hemisphere. (B) Right-hemisphere. (C) Inter-hemisphere. (D) Anterior-region. (E) Posterior-region. (F) Inter-region.

A
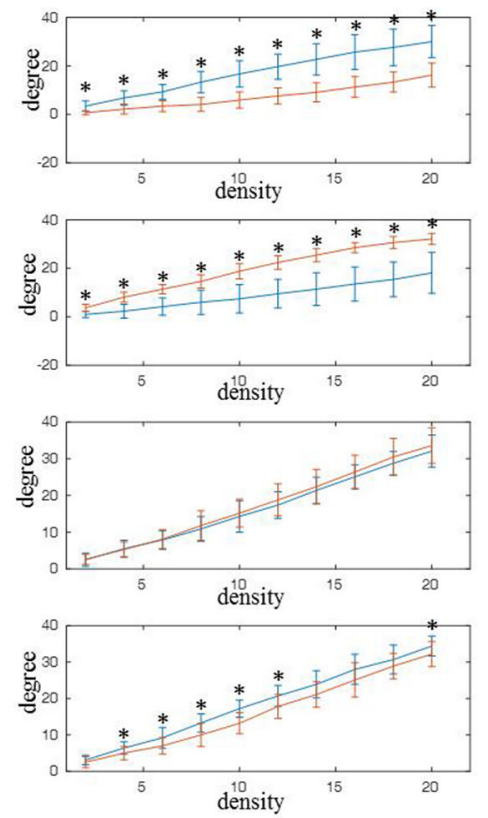

B
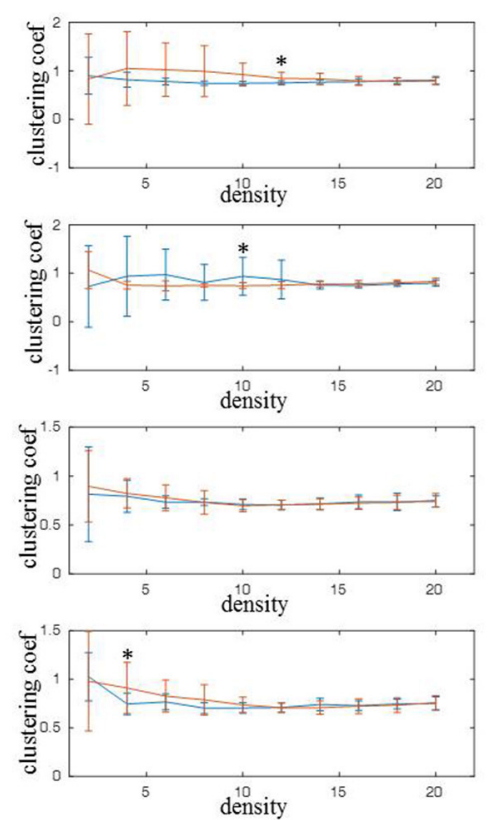

C
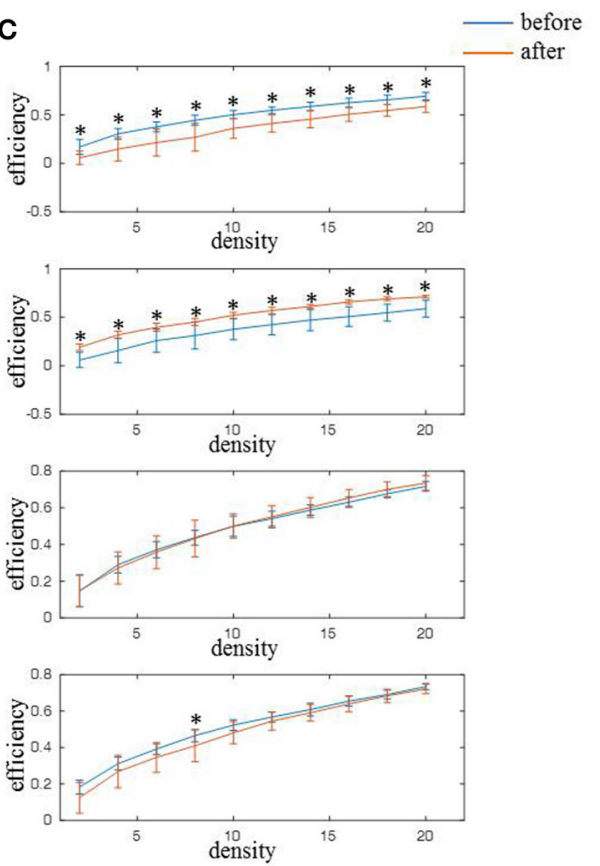

FIGURE 6 | The changes of network characteristics in alpha frequency band for FC4, CP4, C1, and C2 electrodes induced by $r$ TMS-MT (K $=$ from 2 to 20$)\left({ }^{*} p<0.05\right)$. Blue and red lines represent the network characteristics before and after rTMS-MT, respectively. (A) Node degree. (B) Clustering coefficient. (C) Node efficiency.

inter-hemispheres and inter-regions. In other words, the rTMSMT had an obvious impact on long connections of brain regions. Here we showed for the first time that alternations of rTMS-MT could reflect in changes of brain functional connectivity and topological functional organization.

\section{rTMS-MT Improved Motor Performance}

It is important to optimize motor function and improve motor dexterity, especially for post-stroke patients. In recent years, researchers have tested motor performance and corticospinal excitability and found greater behavioral and neurophysiologic 
TABLE 1 | Relationship of functional connectivity and reaction time of Nine-Hole Peg Test for non-dominant hand.

\begin{tabular}{lllll}
\hline & FC4 & CP4 & C2 & C1 \\
\hline correlation & $\mathbf{0 . 7 1 2}$ & 0.371 & 0.594 & 0.672 \\
$P$ & $\mathbf{0 . 0 4 8}$ & 0.365 & 0.121 & 0.068 \\
\hline
\end{tabular}

outcomes induced by rTMS-MT than rTMS or MT alone (Emara et al., 2010; Avenanti et al., 2012; Kakuda et al., 2012, 2016; Lüdemann-Podubecká et al., 2015). In our study, we measured hand dexterity using Nine-Hole Peg Test and also found significant improvement in motor performance after 14-days rTMS-MT (Figure 3), which demonstrated the effectiveness of rTMS-MT in improvement of motor function.

\section{rTMS-MT Modulated the Functional Connectivity in Non-Dominant Hemisphere}

In our study, we evaluated the connectivity changes induced by rTMS-MT, and found it modulated the functional connectivity in non-dominant hemisphere and in a region-specific way (Figure 4). The 14-days MT alone didn't appear those changes (Figure S2) when the significance level was $p<0.005$ which was the same as the rTMS-MT group. Subsequently, we calculated the significant changes induced by MT at a significance level of $p<0.05$ (Figure S3). The results suggested that the functional connectivity of motor cortex in non-dominant hemisphere was also changed. Therefore, the effects of rTMS-MT on functional connectivity were greater than MT alone. In rTMS-MT group, rTMS might play a meaningful role. The changes induced by rTMS-MT were not entirely caused by MT alone.

Besides, the results in our study were inconsistent with the effects of rTMS alone. rTMS could modulated the functional connectivity of widespread cortical networks and activates neural circuits in a non-specific way, although the modulation of rTMS varied as a function of the particular rTMS protocol applied and the frequency band analyzed (Strens et al., 2002; Chen et al., 2003; Oliviero et al., 2003; Fuggetta et al., 2008; Bolognini et al., 2009; Shafi et al., 2014). Especially, the connectivity changes between cortical and cortical regions in stimulated hemisphere were a consistent finding. In our study, we also found widespread changes of functional connectivity induced by rTMSMT. However, the changes in regions induced by rTMS-MT were mainly observed in non-dominant hemisphere (not stimulated hemisphere) related to motor function, especially in premotor and parietal cortices. So, the changes induced by rTMS-MT were not simply caused by rTMS alone.

Taken together, our study represents the first analysis of network-level connectivity changes induced by rTMS-MT. The results suggested that changes induced by rTMS-MT in our study would be in a region-specific way, and might not come from the rTMS or MT alone, and also not linear superposition of rTMS and MT. Motor training could guide the activation of specific neural networks associated with the desired behavior (Bolognini et al., 2009). It was speculated that practice of a motor task might be more effective at using the neural mechanisms sub-serving training-dependent plastic changes if pertinent areas of cortex were facilitated (Ward and Cohen, 2004). The results in our study supported this assumption. rTMS-MT could enhance the functional connectivity induced by MT in a task-specific way.

\section{rTMS-MT Changed the Functional Connectivity and Network Characteristics Related to Motor Function}

We tested changes in functional connectivity induced by rTMS-MT, and found that the alpha band functional connectivity was modulated by $1 \mathrm{~Hz}$ rTMS of the dominant hemisphere combined with MT by the non-dominant hand over 14days. The oscillation at alpha frequency band is the intrinsic rhythms in resting state with eyes closed, which can reflect the state of cortical information processing during motor tasks (Babiloni et al., 2010). Besides, Dubovik et al. (2012) studied changes of synchrony of electrical oscillations in neural networks after stroke, and found the alpha band functional connectivity decreased in ipsilesional central electrodes, which was correlated with motor task performance. Therefore, the functional connectivity at alpha band might be important to improvement of motor function in stroke, and the connection changes induced by rTMS-MT at alpha band oscillation might be the reason of motor improvement.

In our study, the changes of functional connectivity were especially found in the premotor (FC4) and parietal cortices (CP4) in the non-dominant hemisphere. Moreover, the connections of primary motor cortices $(\mathrm{C} 1$ and $\mathrm{C} 2)$ in both hemispheres were also slightly changed (Figures 4A,B). We found a decrease in functional connectivity between premotor and frontal cortices, and an increase between the parietal and frontal cortices (Figure 4C). Motor skill learning involves many processes such as motor planning, motor controlling, spatial orientation, and motor output, and is associated with a variety of cortical activities, including the primary motor, posterior parietal, and premotor cortices, and the functional connectivity between these cortices (Hardwick et al., 2013). Researchers have reported that the connectivity of the resting cortex could predict motor skills, and the connectivity between the frontal and parietal cortices were significantly correlated with the ability to perform motor skills (Wu et al., 2014). Consistent with reported results, we also found that rTMS-MT changed the connectivity between the parietal and frontal cortex in the resting state, along with improvement of motor performance (Figure 4), which was related to the role of the frontal and parietal cortex in motor skills learning (Ma et al., 2011; Wu et al., 2014). Furthermore, the increased connections of premotor cortex were the symbol of inefficient motor system (Ward, 2006). Our study also revealed decreased connections and functional coupling between premotor and frontal cortex, which might imply that rTMS-MT modulated those brain regions to enhance the efficient of the motor system.

We subsequently thresholded the functional connectivity to construct undirected graphs. Threshold can influence the network density. Higher threshold can decrease the number of network connections leading to low network density. In contrast, low threshold can increase the number of network 
connections leading to high network density. In this study, in order to produce the same network density for all subjects before and after rTMS-MT, the threshold was set independently. Because there is no uniform standard for threshold selection, we assessed the network characteristics induced by rTMS-MT in different connectivity degrees ( $\mathrm{K}=$ from 2 to 20 ). The results demonstrated changes in node degree and efficiency of FC4 and CP4 electrodes after rTMS-MT in almost all connectivity degrees (Figure 6). More than other electrodes, the degree and efficiency of FC4 electrode significantly decreased, which suggested the importance of this region became weaker and the information transmission became slower in brain network. Besides, the degree and efficiency of CP4 electrode showed opposite results. These results were consistent with those of functional connectivity. Therefore, rTMS-MT modulated the neural circuits of motor skill learning and increased the efficiency of the motor system in resting state.

Our experimental design was based on the competition model of the two brain hemispheres. According to the purpose of the experimental, rTMS was performed to dominant hemisphere, and non-dominant hand executed the MT. However, we couldn't found remarkable changes of functional connectivity between central and central regions in both hemispheres. We speculated that the changes of brain activity between central and central regions induced by rTMS-MT mightn't be reflected by functional connectivity based on PSI. Grefkes et al. (2008), Grefkes and Fink (2011) studied the changes of connectivity using resting state fMRI in stroke, and didn't found the changes of central-central connectivity, compare with healthy person, which demonstrated the competition between interhemisphere might not be reflected by the central-central connectivity in resting state. Therefore, while we focused on the changes of functional connectivity in resting state induced by rTMS-MT, we couldn't found changes of central-central between interhmisphere. In the course of our experiment, we also assessed the excitability changes of corticospinal tract through testing the motor evoked potentials (MEP), the result was shown in Supplementary Material 2 (Figure S6). We found the MEP amplitudes of non-dominant hand increased and the dominant hand didn't change, which indicated change of central and central regions and reflected the competition model to a certain degree.

\section{rTMS-MT Could Modulate Network Topology}

In our study, although rTMS, and MT were applied to the left motor cortex (dominant hemisphere) and left hand (nondominant hemisphere), respectively, we found changes in connections mainly occurred within inter-hemisphere at almost all network densities, rather than left or right hemisphere (Figure 5). This was suggestion that the rTMS-MT increased the connections and the information transmission between inter-hemisphere. It has been reported that homologous and heterologous regions of inter-hemispheric regions were connected through a large number of nerve fibers in the corpus callosum, which supported information transmission and functional integration of the two hemispheres (Jing and Takigawa, 2000; Paul et al., 2007). Therefore, the increasing connections were consistent with the presence of significant transcallosal anatomic connectivity between homologous areas.

In addition, the increased connections within interhemisphere induced by rTMS-MT might be a critical factor for improvement of motor function in stroke. Previous studies utilizing a variety of neuroimaging techniques had demonstrated that there were widespread cortical networks abnormalities after stroke, which were reflected in not only in lesion regions but also among the interactions among cortical areas distant from the lesion (Honey and Sporns, 2008; Carter et al., 2010; Grefkes et al., 2010; Wang et al., 2010). Furthermore, the consistent finding was that inhibition of inter-hemisphere was changed in patients with motor dysfunctions, and the improvement of motor performance was shown to be correlated with restitution of inter-hemispheric connectivity (Ward and Cohen, 2004; He et al., 2007; Carter et al., 2010; van Meer et al., 2010). It was helpful for recovery of motor function to enhanced inter-hemispheric connectivity (Grefkes et al., 2010). Therefore, the effects of rTMS-MT on inter-hemispheric connectivity might be another reason for improvement of motor performance.

\section{LIMITATIONS}

There were certain limitations with our study. First, the number of subjects was small (10 subjects were included in the final analysis) and there was no blank control group. Future studies with more subjects and/or a group of blank control subjects could help provide greater insight into resting state network changes associated with the effects of rTMS-MT. Furthermore, all subjects in this study were healthy people. Although, motor performance enhancement is important for healthy individuals, it is more meaningful for studies on patients with motor dysfunctions. Moreover, brain activity influenced by rTMS-MT on patients might be different from that on the healthy person, so it is necessary in the next step to study the effect of rTMS-MT on functional connectivity of patients, especially people with stroke. An additional consideration is that the design of this study did not allow dissection of effects induced by each intervention (i.e., rTMS or MT) on functional connectivity. In our study, all volunteers are all healthy. The tasks were selected to improve the hand dexterity. We didn't test other function, such as hand grip power and pinch power and so on, which might be a further limitation of this study. Notwithstanding these limitations, the results in this study were helpful in understanding the modulation of rTMS-MT on functional connectivity. Meanwhile, the methods in this study could in future be used to evaluate changes of brain activities induced by rTMS-MT in stroke patients.

\section{CONCLUSION}

In summary, our findings suggest that rTMS-MT could improve motor function and induce significant changes in functional connectivity and network characteristics in regions related to motor functions. The network analysis techniques might be a promising method to study how changes of information integration occur between regions induced by rTMS-MT. The 
findings in this paper could be helpful in understanding the impact of rTMS-MT on brain activity. The methods and results in this study could be taken as reference for future research of the effects of rTMS-MT in stroke patients.

\section{AUTHOR CONTRIBUTIONS}

J-NJ, YL, and FJ were responsible for the design of the work, and completed the experiment for acquiring the EEG data. After that, J-NJ, XW, and Z-PL completed the analysis and the interpretation of the data. All authors participated in drafting the manuscript. J-NJ, Z-PL, and TY were responsible for revising the important intellectual content involved in the article and approved the final version of the article.

\section{REFERENCES}

Angelini, L., de Tommaso, M., Guido, M., Hu, K., Ivanov, P. C., Marinazzo, D., et al. (2004). Steady-state visual evoked potentials and phase synchronization in migraine patients. Phys. Rev. Lett. 93:38103. doi: 10.1103/PhysRevLett.93.038103

Avenanti, A., Coccia, M., Ladavas, E., Provinciali, L., and Ceravolo, M. G. (2012). Low-frequency rTMS promotes use-dependent motor plasticity inchronic stroke: a randomized trial. Neurology 78, 256-264. doi: 10.1212/WNL.0b013e3182436558

Babiloni, C., Marzano, N., Iacoboni, M., Infarinato, F., Aschieri, P., Buffo, P., et al. (2010). Resting state cortical rhythms in athletes: a high-resolution EEG study. Brain Res. Bull. 81, 149-156. doi: 10.1016/j.brainresbull.2009.10.014

Baeken, C., Schrijvers, D. L., Sabbe, B. G., Vanderhasselt, M. A., and De Raedt, R. (2012). Impact of one HF-rTMS session on fine motor function in righthanded healthy female subjects: a comparison of stimulation over the left versus the right dorsolateral prefrontal cortex. Neuropsychobiology 65, 96-102. doi: $10.1159 / 000329699$

Bolognini, N., Pascual-Leone, A., and Fregni, F. (2009). Using non-invasive brain stimulation to augment motor training-induced plasticity. J. Neuroeng. Rehabil. 6:8. doi: 10.1186/1743-0003-6-8

Brodie, S. M., Meehan, S., Borich, M. R., and Boyd, L. A. (2014). $5 \mathrm{~Hz}$ repetitive transcranial magnetic stimulation over the ipsilesional sensory cortex enhances motor learning after stroke. Front. Hum. Neurosci. 8:143. doi: $10.3389 /$ fnhum. 2014.00143

Carter, A. R., Astafiev, S. V., Lang, C. E., Connor, L. T., Rengachary, J., Strube, M. J., et al. (2010). Resting interhemispheric functional magnetic resonance imaging connectivity predicts performance after stroke. Ann. Neurol. 67, 365-375. doi: $10.1002 /$ ana. 21905

Chen, W. H., Mima, T., Siebner, H. R., Oga, T., Hara, H., Satow, T., et al. (2003). Low-frequency rTMS over lateral premotor cortex induces lasting changes in regional activation and functional coupling of cortical motor areas. Clin. Neurophysiol. 114, 1628-1637. doi: 10.1016/S1388-2457(03) 00063-4

Conforto, A. B., Anjos, S. M., Saposnik, G., Mello, E. A., Nagaya, E. M., Santos, W. Jr., et al. (2012). Transcranial magnetic stimulation in mild to severe hemiparesis early after stroke: a proof of principle and novel approach to improve motor function. J. Neurol. 259, 1399-1405. doi: 10.1007/s00415-011-6364-7

D’Agata, F., Peila, E., Cicerale, A., Caglio, M. M., Caroppo, P., Vighetti, S. et al. (2016). Cognitive and neurophysiological effects of non-invasive brain stimulation in stroke patients after motor rehabilitation. Front. Behav. Neurosci. 10:135. doi: 10.3389/fnbeh.2016.00135

David, O., Cosmelli, D., and Friston, K. J. (2004). Evaluation of different measures of functional connectivity using a neural mass model. Neuroimage 21, 659-673. doi: 10.1016/j.neuroimage.2003.10.006

De Vico Fallani, F., Clausi, S., Leggio, M., Chavez, M., Valencia, M., Maglione, A. G., et al. (2017). Interhemispheric connectivity characterizes cortical

\section{FUNDING}

This research was supported by CAMS Initiative for Innovative Medicine (No. 2016-I2M-1004), Special Funds of the key technologies $\mathrm{R}$ \& $\mathrm{D}$ program of Tianjin, China (No. 17YFZCSY00730), National Natural Science Foundation of China (No. 81772003), and PUMC YouthFund (No. 3332016104).

\section{SUPPLEMENTARY MATERIAL}

The Supplementary Material for this article can be found online at: https://www.frontiersin.org/articles/10.3389/fnbeh. 2017.00234/full\#supplementary-material

reorganization in motor-related networks after cerebellar lesions. Cerebellum 16, 358-375. doi: 10.1007/s12311-016-0811-z

Dubovik, S., Pignat, J. M., Ptak, R., Aboulafia, T., Allet, L., Gillabert, N., et al. (2012). The behavioral significance of coherent resting-state oscillations after stroke. Neuroimage 61:249. doi: 10.1016/j.neuroimage.2012.03.024

Emara, T. H., Moustafa, R. R., Elnahas, N. M., Elganzoury, A. M., Abdo, T. A., Mohamed, S. A., et al. (2010). Repetitive transcranial magnetic stimulation at $1 \mathrm{~Hz}$ and $5 \mathrm{~Hz}$ produces sustained improvement in motor function and disability after ischaemic stroke. Eur. J. Neurol. 17, 1203-1209. doi: 10.1111/j.1468-1331.2010.03000.x

Farzan, F., Barr, M. S., Levinson, A. J., Chen, R., Wong, W., Fitzgerald, P. B., et al. (2010). Reliability of long interval cortical inhibition in healthy human subjects: a TMS-EEG study. J. Neurophysiol. 104, 1339-1346. doi: 10.1152/jn.00279.2010

Fox, M. D., and Raichle, M. E. (2007). Spontaneous fluctuations in brain activity observed with functional magnetic resonance imaging. Nat. Rev. Neurosci. 8, 700-711. doi: 10.1038/nrn2201

Fregni, F., Boggio, P. S., Valle, A. C., Rocha, R. R., Duarte, J., Ferreira, M. J., et al. (2006). A sham-controlled trial of a 5-day course of repetitive transcranial magnetic stimulation of the unaffected hemisphere in stroke patients. Stroke 37, 2115-2122. doi: 10.1161/01.STR.0000231390.58967.6b

Fuggetta, G., Pavone, E. F., Fiaschi, A., and Manganotti, P. (2008). Acute modulation of cortical oscillatory activities during short trains of highfrequency repetitive transcranial magnetic stimulation of the human motor cortex: a combined EEG and TMS study. Hum. Brain Mapp. 29, 1-13. doi: 10.1002/hbm.20371

Grefkes, C., and Fink, G. R. (2011). Reorganization of cerebral networks after stroke: new insights from neuroimaging with connectivity approaches. Brain 134, 1264-1276. doi: 10.1093/brain/awr033

Grefkes, C., Nowak, D. A., Eickhoff, S. B., Dafotakis, M., Küst, J., Karbe, H., et al. (2008). Cortical connectivity after subcortical stroke assessed with functional magnetic resonance imaging. Ann. Neurol. 63, 236-246. doi: 10.1002/ana.21228

Grefkes, C., Nowak, D. A., Wang, L. E., Dafotakis, M., Eickhoff, S. B., and Fink, G. R. (2010). Modulating cortical connectivity in stroke patients by rTMS assessed with fMRI and dynamic causal modelling. Neuroimage 50, 233-242. doi: 10.1016/j.neuroimage.2009.12.029

Hardwick, R. M., Rottschy, C., Miall, R. C., and Eickhoff, S. B. (2013). A quantitative meta-analysis and review of motor learning in the human brain. Neuroimage 67, 283-297. doi: 10.1016/j.neuroimage.2012.11.020

He, B. J., Snyder, A. Z., Vincent, J. L., Epstein, A., Shulman, G. L., and Corbetta, M. (2007). Breakdown of functional connectivity in frontoparietal networks underlies behavioral deficits in spatial neglect. Neuron 53, 905-918. doi: 10.1016/j.neuron.2007.02.013

Higgins, J., Salbach, N. M., Wood-Dauphinee, S., Richards, C. L., Côté, R., and Mayo, N. E. (2006). The effect of a task-oriented intervention on arm function in people with stroke: a randomized controlled trial. Clin. Rehabil. 20, 296-310. doi: 10.1191/0269215505cr943oa

Honey, C. J., and Sporns, O. (2008). Dynamical consequences of lesions in cortical networks. Hum. Brain Mapp. 29, 802-809. doi: 10.1002/hbm.20579 
Ito, J., Nikolaev, A. R., and Leeuwen, C. (2005). Spatial and temporal structure of phase synchronization of spontaneous alpha EEG activity. Biol. Cybern. 92, 54-60. doi: 10.1007/s00422-004-0533-z

Jing, H., and Takigawa, M. (2000). Observation of EEG coherence after repetitive transcranial magnetic stimulation. Clin. Neurophysiol. 111, 1620-1631. doi: 10.1016/S1388-2457(00)00357-6

Kakuda, W., Abo, M., Sasanuma, J., Shimizu, M., Okamoto, T., Kimura, C., et al. (2016). Combination protocol of low-frequency rTMS and intensive occupational therapy for post-stroke upper limb hemiparesis: a 6-year experience of more than 1700 Japanese patients. Trans. Stroke Res. 7, 172-179. doi: 10.1007/s12975-016-0456-8

Kakuda, W., Abo, M., Shimizu, M., Sasanuma, J., Okamoto, T., Yokoi, A., et al. (2012). A multi-center study on low-frequency rTMS combined with intensive occupational therapy for upper limb hemiparesis in post-stroke patients. J. Neuroeng. Rehabil. 9:4. doi: 10.1186/1743-0003-9-4

Kondo, T., Kakuda, W., Yamada, N., Shimizu, M., and Abo, M. (2015). Effects of repetitive transcranial magnetic stimulation and intensive occupational therapy on motor neuron excitability in post stroke hemiparetic patients: a neurophysiological investigation using F-wave parameters. Int. J. Neurosci. 125, 25-31. doi: 10.3109/00207454.2014.897706

Lüdemann-Podubecká, J., Bösl, K., Theilig, S., Wiederer, R., and Nowak, D. A. (2015). The effectiveness of $1 \mathrm{~Hz}$ rTMS over the primary motor area of the unaffected hemisphere to improve hand function after stroke depends on hemispheric dominance. Brain Stimul. 8, 823-830. doi: 10.1016/j.brs.2015.02.004

Ma, L., Narayana, S., Robin, D. A., Fox, P. T., and Xiong, J. (2011). Changes occur in resting state network of motor system during 4 weeks of motor skill learning. Neuroimage 58, 226-233. doi: 10.1016/j.neuroimage.2011. 06.014

van Meer, M. P., van der Marel, K., Wang, K., Otte, W. M., El Bouazati, S., Roeling, T. A., et al. (2010). Recovery of sensorimotor function after experimental stroke correlates with restoration of resting-state interhemispheric functional connectivity. J. Neurosci. 30, 3964-3972. doi: 10.1523/JNEUROSCI.5709-09.2010

Oliviero, A., Strens, L. H., Di Lazzaro, V., Tonali, P. A., and Brown, P. (2003). Persistent effects of high frequency repetitive TMS on the coupling between motor areas in the human. Exp. Brain Res. 149, 107-113. doi: 10.1007/s00221-002-1344-x

Paul, L. K., Brown, W. S., Adolphs, R., Tyszka, J. M., Richards, L. J., Mukherjee, P., et al. (2007). Agenesis of the corpus callosum: genetic developmental and functional aspects of connectivity. Nat. Rev. Neurosci. 8, 287-299. doi: $10.1038 / \mathrm{nrn} 2107$

Plewnia, C., Rilk, A. J., Soekadar, S. R., Arfeller, C., Huber, H. S., Sauseng, P., et al. (2008). Enhancement of long-range EEG coherence by synchronous bifocal transcranial magnetic stimulation. Eur. J. Neurosci. 27, 1577-1583. doi: 10.1111/j.1460-9568.2008.06124.x

Quian Quiroga, R., Kraskov, A., Kreuz, T., and Grassberger, P. (2002). Performance of different synchronization measures in real data: a case study on electroencephalographic signals. Phys. Rev. E 65:41903. doi: 10.1103/PhysRevE.65.041903

Quiroga, R. Q., Arnhold, J., and Grassberger, P. (2000). Learning driver-response relationships from synchronization patterns. Phys. Rev. E 61, 5142-5148. doi: 10.1103/PhysRevE.61.5142

Shafi, M. M., Brandon Westover, M., Oberman, L., Cash, S. S., and Pascual-Leone, A. (2014). Modulation of EEG functional connectivity networks in subjects undergoing repetitive transcranial magnetic stimulation. Brain Topogr. 27, 172-191. doi: 10.1007/s10548-013-0277-y

Sporns, O., Chialvo, D. R., Kaiser, M., and Hilgetag, C. C. (2004). Organization, development and function of complex brain networks. Trends Cogn. Sci. 8, 418-425. doi: 10.1016/j.tics.2004.07.008

Stam, C. J., and Reijneveld, J. C. (2007). Graph theoretical analysis of complex networks in the brain. Nonlin. Biomed. Phys. 1:3. doi: 10.1186/1753-4631-1-3

Stam, C. J., Jones, B. F., Nolte, G., Breakspear, M., and Scheltens, P. (2007a). Smallworld networks and functional connectivity in Alzheimer's disease. Cereb. Cortex 17, 92-99. doi: 10.1093/cercor/bhj127
Stam, C. J., de Haan, W., Daffertshofer, A., Jones, B. F., Manshanen, I., van Cappellen van Walsum, A. M., et al. (2007b). Graph theoretical analysis of magnetoencephalographic functional connectivity in Alzheimer's disease. Brain 132, 213-224. doi: 10.1093/brain/ awn 262

Stern, P. (2013). The heavily connected brain. Science 342, 577-585. doi: 10.1126/science.342.6158.577

Strens, L. H., Oliviero, A., Bloem, B. R., Gerschlager, W., Rothwell, J. C., and Brown, P. (2002). The effects of subthreshold $1 \mathrm{~Hz}$ repetitive TMS on corticocortical and interhemispheric coherence. Clin. Neurophysiol. 113, 1279-1285. doi: 10.1016/S1388-2457(02)00151-7

Takekawa, T., Kakuda, W., Uchiyama, M., Ikegaya, M., and Abo, M. (2014). Brain perfusion and upper limb motor function: a pilot study on the correlation between evolution of asymmetry in cerebral blood flow and improvement in Fugl-meyer assessment score after rTMS in chronic post-stroke patients. $J$. Neuroradiol. 41, 177-183. doi: 10.1016/j.neurad.2013.06.006

Thut, G., and Pascual-Leone, A. (2010). A review of combined TMS-EEG studies to characterize lasting effects of repetitive TMS and assess their usefulness in cognitive and clinical neuroscience. Brain Topogr. 22, 219-232. doi: 10.1007/s10548-009-0115-4

Turriziani, P., Smirni, D., Zappalà, G.,Magnano, G. R., Oliveri, M., and Cipolotti, L. (2012). Enhancing memory performance with rTMS in healthy subjects and individuals with Mild cognitive impairment: the role of the right dorsolateral prefrontal cortex. Front. Hum. Neurosci. 6:62. doi: 10.3389/fnhum.2012.00062

Van Dijk, K. R., Hedden, T., Venkataraman, A., Evans, K. C., Lazar, S. W., and Buckner, R. L. (2010). Intrinsic functional connectivity as a tool for human connectomics: theory, properties, and optimization. J. Neurophysiol. 103, 297-321. doi: 10.1152/jn.00783.2009

Van, J. H., Snels, I. A., Beckerman, H., Lankhorst, G. L., and Wagenaar, R. C. (2001). Exercise therapy for arm function in stroke patients: a systematic review of randomized controlled trails. Clin. Rehabil. 15, 20-31. doi: 10.1191/026921501677557755

Varela, F., Lachaux, J. P., Rodriguez, E., and Martinernie, J. (2001). The brainweb: phase synchronization and large-scale integration. Nat. Rev. Neurosci. 2, 229-239. doi: 10.1038/35067550

Wang, L., Yu, C., Chen, H., Qin, W., He, Y., Fan, F., et al. (2010). Dynamic functional reorganization of the motor execution network after stroke. Brain 133, 1224-1238. doi: 10.1093/brain/awq043

Ward, N. S., and Cohen, L. G. (2004). Mechanisms underlying recovery of motor function after stroke. Arch. Neurol. 61, 1844-1848. doi: 10.1001/archneur.61.12.1844

Ward, N. S. (2006). Compensatory mechanisms in the aging motor system. Ageing Res. Rev. 5, 239-254. doi: 10.1016/j.arr.2006.04.003

Wu, J., Srinivasan, R., Kaur, A., and Cramer, S. C. (2014). Resting-state cortical connectivity predicts motor skill acquisition. Neuroimage 91, 84-90. doi: 10.1016/j.neuroimage.2014.01.026

Wu, W., Sun, J., Jin, Z., Guo, X., Qiu, Y., Zhu, Y., et al. (2011). Impaired neuronal synchrony after focal ischemic stroke in elderly patients. Clin. Neurophysiol. 122, 21-26. doi: 10.1016/j.clinph.2010.06.003

Youssofzadeh, V., Zanotto, D., Wong-Lin, K., Agrawal, S. K., and Prasad, G. (2016). Directed functional connectivity in fronto-centroparietal circuit correlates with motor adaptation in gait training. IEEE Trans. Neural Syst. Rehabil. Eng. 24, 1265-1275. doi: 10.1109/TNSRE.2016. 2551642

Conflict of Interest Statement: The authors declare that the research was conducted in the absence of any commercial or financial relationships that could be construed as a potential conflict of interest.

Copyright (c) 2017 Jin, Wang, Li, Jin, Liu and Yin. This is an open-access article distributed under the terms of the Creative Commons Attribution License (CC BY). The use, distribution or reproduction in other forums is permitted, provided the original author(s) or licensor are credited and that the original publication in this journal is cited, in accordance with accepted academic practice. No use, distribution or reproduction is permitted which does not comply with these terms. 